1 Assessing the ability of MODIS EVI to estimate terrestrial ecosystem gross primary

$4 \quad$ Hao $\mathrm{Shi}^{1}$, Longhui $\mathrm{Li}^{1}{ }^{1}$, , Derek Eamus ${ }^{2}$, Alfredo Huete ${ }^{1}$, James Cleverly $^{2}$, Xin Tian $^{3}$, Qiang

$5 \mathrm{Yu}^{1}$, Shaoqiang $\mathrm{Wang}^{4}$, Leonardo Montagnani ${ }^{5}$, Vincenzo Magliulo ${ }^{6}$, Eyal Rotenberg ${ }^{7}$, 6 Marian Pavelka ${ }^{8}$, Arnaud Carrara $^{9}$

$8{ }^{1}$ School of Life Sciences, University of Technology Sydney, Sydney 2000, NSW, Australia

$9{ }^{2}$ Australian Supersite Network, University of Technology Sydney, Sydney 2000, NSW, 10 Australia

$11{ }^{3}$ Research Institute of Forest Resource Information Techniques, Chinese Academy of 12 Forestry, Beijing 100091, People's Republic of China

${ }^{4}$ Key Laboratory of Ecosystem Network Observation and Modeling, Institute of Geographic

Sciences and Natural Resources Research, Chinese Academy of Sciences, Beijing 100101,

People's Republic of China

${ }^{5}$ Faculty of Science and Technology, Free University of Bolzano, Piazza Università 1, 39100,

17 Italy

${ }^{6}$ CNR Institute for Agricultural and Forest Systems, Via Patacca 85, 80056 Ercolano (Napoli), Italy

${ }^{7}$ Department of Environmental Sciences and Energy Research, Weizmann Institute of

Science, Rehovot 76100, Israel

${ }^{8}$ Laboratory of Plants Ecological Physiology, Institute of Systems Biology and Ecology AS 
${ }^{9}$ Fundación Centro de Estudios Ambientales del Mediterráneo (CEAM), Charles Darwin 14, Parc Tecnològic, 46980 Paterna, Spain

\section{*Corresponding address:}

Plant Functional Biology and Climate Change Cluster

University of Technology, Sydney

PO Box 123, Sydney, NSW 2007, Australia

Tel: +61 29514 8349;

Fax: +61 29514 4003;

Email: Longhui.Li@uts.edu.au

\section{Abstract}

Terrestrial ecosystem gross primary production (GPP) is the largest component in the global carbon cycle. The enhanced vegetation index (EVI) has been proven to be strongly correlated with annual GPP within several biomes. However, the annual GPP-EVI relationship and associated environmental regulations have not yet been comprehensively investigated across biomes at the global scale. Here we explored relationships between annual integrated EVI (iEVI) and annual GPP observed at 155 flux sites, where GPP was predicted with a log-log model: $\ln (G P P)=a \times \ln (i E V I)+b$. iEVI was computed from MODIS monthly EVI products following removal of values affected by snow or cold temperature and without calculating growing season duration. Through categorisation of flux sites into 12 land cover types, the ability of iEVI to estimate GPP was considerably improved ( $R^{2}$ from 0.62 to 0.74 , RMSE from 454.7 to $368.2 \mathrm{~g} \mathrm{C} \mathrm{m}^{-2} \mathrm{yr}^{-1}$ ). The biome-specific GPP-iEVI formulae generally showed 
47 a consistent performance in comparison to a global benchmarking dataset $\left(R^{2}=0.79\right.$, RMSE $\left.48=387.8 \mathrm{~g} \mathrm{C} \mathrm{m}^{-2} \mathrm{yr}^{-1}\right)$. Specifically, iEVI performed better in cropland regions with high productivity but poorer in forests. The ability of iEVI in estimating GPP was better in deciduous biomes (except deciduous broadleaf forest) than in evergreen due to the large seasonal signal in iEVI in deciduous biomes. Likewise, GPP estimated from iEVI was in a closer agreement to global benchmarks at mid and high-latitudes, where deciduous biomes are more common and cloud cover has a smaller effect on remote sensing retrievals. Across biomes, a significant and negative correlation $\left(R^{2}=0.37, p<0.05\right)$ was observed between the strength $\left(R^{2}\right)$ of GPP-iEVI relationships and mean annual maximum leaf area index $\left(\mathrm{LAI}_{\max }\right)$, and the relationship between the strength and mean annual precipitation followed a similar trend. $\mathrm{LAI}_{\max }$ also revealed a scaling effect on GPP-iEVI relationships. Our results suggest that iEVI provides a very simple but robust approach to estimate spatial patterns of global annual GPP whereas its effect is comparable to various light-use-efficiency and data-driven models. The impact of vegetation structure on accuracy and sensitivity of EVI in estimating spatial GPP provides valuable clues to improve EVI-based models.

Keywords: Remote sensing; MODIS; Enhanced vegetation index; Gross primary production; Land cover types; Leaf area index

\section{Introduction}

Terrestrial gross primary production (GPP) is the amount of carbon captured from the atmosphere through vegetation photosynthesis (Beer et al., 2010). Vegetation GPP is a key component of the terrestrial carbon balance and is of fundamental importance to human society because plants provide food, fiber and wood supply and also contribute to the 
production of environmental conditions suitable for human habitation (Melillo et al., 1993; Xiao et al., 2005; Zhao et al., 2005). Therefore, continuous monitoring and accurate estimation of GPP is required to ensure the long term security of terrestrial ecosystem services and to address issues pertaining to the global carbon cycle including determination of the size of the terrestrial carbon sink, prediction of vegetation dynamics, and management of forests and grasslands (Ciais et al., 2005; Ma et al., 2013; Sims et al., 2006b).

GPP can be calculated as the sum of vegetation assimilated carbon flux, partitioned from net carbon exchange measured at eddy covariance (EC) tower sites (Baldocchi et al., 2001; Reichstein et al., 2007), but such observations are limited, both temporally and spatially. Remote sensing technique provides a promising approach to overcome these limitations. Various diagnostic models taking advantage of spatially extensive remote sensing and meteorological data have been developed to estimate GPP across stand-to-global scales for a relatively long period (e.g., Jung et al., 2008; Running et al., 2004; Sims et al., 2008; Xiao et al., 2005). These models can be generally partitioned into three categories: light-useefficiency (LUE) models, machine learning algorithms and simple empirical models (Verma et al., 2014). The LUE theory was first proposed by Monteith (1972), in which GPP is generally represented as the product of LUE, photosynthetically active radiation (PAR), the fraction of PAR absorbed by vegetation (fAPAR), and environmental scalars. fAPAR is a strong function of vegetation greenness, as measured by vegetation indices (VIs), such as the normalized difference vegetation index (NDVI; e.g., Goward and Huemmrich, 1992) and the enhanced vegetation index (EVI; e.g., Xiao et al., 2004a; Xiao et al., 2004b). However, it is difficult to estimate LUE, which varies among plant functional types, and can be downregulated by temperature, soil water content, vapour pressure deficit (VPD), and leaf phenology (Xiao et al., 2005). Another deficiency of LUE models is the coarse resolution of climate inputs, which are often only available at a large scale. This may introduce significant 
errors to estimations of GPP (Heinsch et al., 2006; Zhao et al., 2005) and hinder the acquisition of fine-resolution GPP estimates at large scales. Machine learning algorithms, such as artificial neural networks (Papale and Valentini, 2003), support vector machines (Yang et al., 2007), and model tree ensembles (Jung et al., 2009), predict GPP based on the non-functional patterns extracted in training data set. Obviously, the accuracy of machine learning algorithms relies on the abundance and representativeness of input information including remote sensed vegetation properties, meteorological, and land cover data (Jung et al., 2011). Therefore, the use of machine learning algorithms is also limited by the coarse resolution of meteorological data. Moreover, in many cases machine learning algorithms show no better performance than LUE models in specific ecosystems (e.g., Yang et al., 2007). Consequently, simple empirical models utilizing remote sensing proxies of vegetation photosynthesis activity (with or without meteorological data) gain consistent interest in estimating both spatial and temporal variations of GPP (e.g., Jung et al., 2008; Rahman et al., 2005; Sims et al., 2006b).

The growing season NDVI and EVI show strong relationships with vegetation production over one or two week intervals (e.g., Mao et al., 2014; Rahman et al., 2005; Sims et al., 2006a; Sims et al., 2006b; Wylie et al., 2003). Vegetation indices per se are transformations of two or more spectral bands to enhance the signal derived from vegetation properties (Huete et al., 2002). Both NDVI and EVI employ surface bidirectional reflectances of red and near-infrared spectral bands that are sensitive to leaf chlorophyll content (Huete et al., 2002), which converts light to energy consumed by photosynthesis. NDVI is limited due to its saturation over dense vegetation and large sensitivity to canopy background brightness (Huete et al., 2002), whereas EVI can improve performance in regions of high biomass through a decoupling of the canopy and background signals and a reduction in the influence of atmospheric conditions using a blue spectral reflectance (Huete et al., 2002). This makes 
EVI more responsive to canopy structural variations and thus EVI is better correlated with GPP than NDVI in evergreen (Xiao et al., 2004a) and deciduous (Xiao et al., 2004b) forests as well as in croplands (Xiao et al., 2005). Compared to LUE models, the growing season EVI or EVI-based models (e.g., Temperature-Greenness model; Sims et al., 2008) provide a comparable or better estimation of GPP at both the 16-day (Sims et al., 2008; Sims et al., 2006b) and annual (Verma et al., 2014) time-scales. As well as EVI, cumulative growing season fAPAR with separate functions for herbaceous plants, evergreen forests and all other vegetation types has been used to predict annual GPP in Europe (Jung et al., 2008). The disadvantage of selecting fAPAR against EVI is subtle: fAPAR consists of fractional absorbance of PAR absorbed by both chlorophyll and by non-photosynthetic pigments (Zhang et al., 2005), while EVI is much closer to the fraction of PAR absorbed by chlorophyll. Moreover, fAPAR shows no significant correlation with GPP in deciduous broadleaf forests (Jung et al., 2008). Therefore, the use of EVI should be favored over fAPAR in correlating to GPP. However, current studies on EVI-GPP relationships or EVIbased models have been focused within only a limited number of biomes and these EVIbased models generally need to compute the start and end or the length of the growing season period (Jung et al., 2008; Sims et al., 2008; Sims et al., 2006b; Verma et al., 2014), which constitutes an extra source of uncertainty. Simultaneously, environmental influences on the ability of EVI to estimate GPP across a wide spectrum of biomes have not yet been investigated (Sims et al., 2006b; Sjöström et al., 2011).

In this study, we used the annual integral of MODIS EVI (iEVI), which only needs removal of those values that have been affected by cold temperature or snow and subtracting the soil background signal, to regress with annual eddy covariance measured GPP across 12 land cover types. The developed set of formulae were then applied at the global scale and 
robustness of $\mathrm{iEVI}$, thereby determining whether iEVI can serve as a reference for other GPP models over a fine-to-coarse resolution. The impacts of environmental conditions on iEVI in estimating GPP were further investigated across biomes, to improve our understanding of the underlying mechanistic processes that differentiate responses of vegetation photosynthetic activity to remote sensing spectral measurements among biomes.

\section{Data and Methods}

\subsection{Eddy covariance and meteorological data}

The eddy covariance method is a micrometeorological technique that directly measures net carbon, water and energy fluxes across a horizontal plane between vegetation canopies and the atmosphere (Aubinet et al., 2000; Baldocchi et al., 2001). In the present study a total of 155 sites (Supplementary Table S1) were selected, consisting of 624 site-years of data and representing a worldwide spectrum of biomes and climate regimes with excellent coverage in North America, Eurasia and Oceania (Table 1, Fig. 1; Baldocchi, 2008; Baldocchi et al., 2001; Wang and Dickinson, 2012).

The flux data were obtained from three sources: (1) a small fraction (mainly high-latitude and wetland sites) was collected directly from published studies, which only included annual values of flux and meteorological forcing; (2) a larger fraction was contributed directly from participating site researchers; and (3) the majority were from FLUXNET level 2 or level 4 products that were downloaded from the database. Of the latter two categories, only siteyears with small gaps (i.e., individual gaps in NEE of less than 5\% of the entire annual record) were selected except in certain ecosystems of the boreal region where only growing season data were available. Carbon and water fluxes and meteorological variables in all selected siteyears were then processed through gap-filling and flux partitioning routines. If the principal 
investigator at each site had already performed these processes, the already gap-filled and partitioned GPP dataset was aggregated from a half-hourly time-step to an annual time-scale $\left(\mathrm{GPP}_{\mathrm{EC}}\right)$. Otherwise, half-hourly GPP derived using one of the two FLUXNET standard methods, either the marginal distribution sampling (MDS, a local method; Reichstein et al., 2005) or a feed-forward artificial neural network (ANN, trained on an annual dataset; Papale and Valentini, 2003), were obtained from the FLUXNET products to calculate annual GPP. Both partitioning methods show good performance according to previous studies (Papale and Valentini, 2003; Reichstein et al., 2005). For sites with neither investigator's decomposition nor standardized flux partitioning, the publicly available online MDS tool (http://www.bgcjena.mpg.de/ MDIwork/eddyproc/index.php; Reichstein et al., 2005) was used to gap-fill and partition NEE. The derived half-hourly GPP, temperature, precipitation and vapour pressure deficit (VPD) of all site-years were screened for outliers and linearly interpolated in bins representing the measurement hour of the day before aggregation into the annual scale.

Table 1. Summary of number of sites and site-years used for each biome. CNM: cropland/natural vegetation mosaic; CRO: croplands; CSH: closed shrublands; DBF: deciduous broadleaf forest; DNF: deciduous needle-leaf forest; EBF: evergreen broadleaf forest; ENF: evergreen needle-leaf forest; GRA: grasslands; MF: mixed forest; OSH: open shrublands; SAV: savannas; WET: permanent wetlands; WSA: woody savannas.

Biome $\quad$ CRO CSH DBF DNF EBF ENF GRA MF OSH SAV WET WSA

\begin{tabular}{lcccccccccccc}
\hline Sites & 16 & 4 & 18 & 4 & 13 & 40 & 24 & 8 & 7 & 7 & 9 & 5 \\
& & & & & & & & & & & & \\
\hline Site-years & 61 & 11 & 83 & 6 & 55 & 190 & 76 & 28 & 21 & 24 & 34 & 35
\end{tabular}




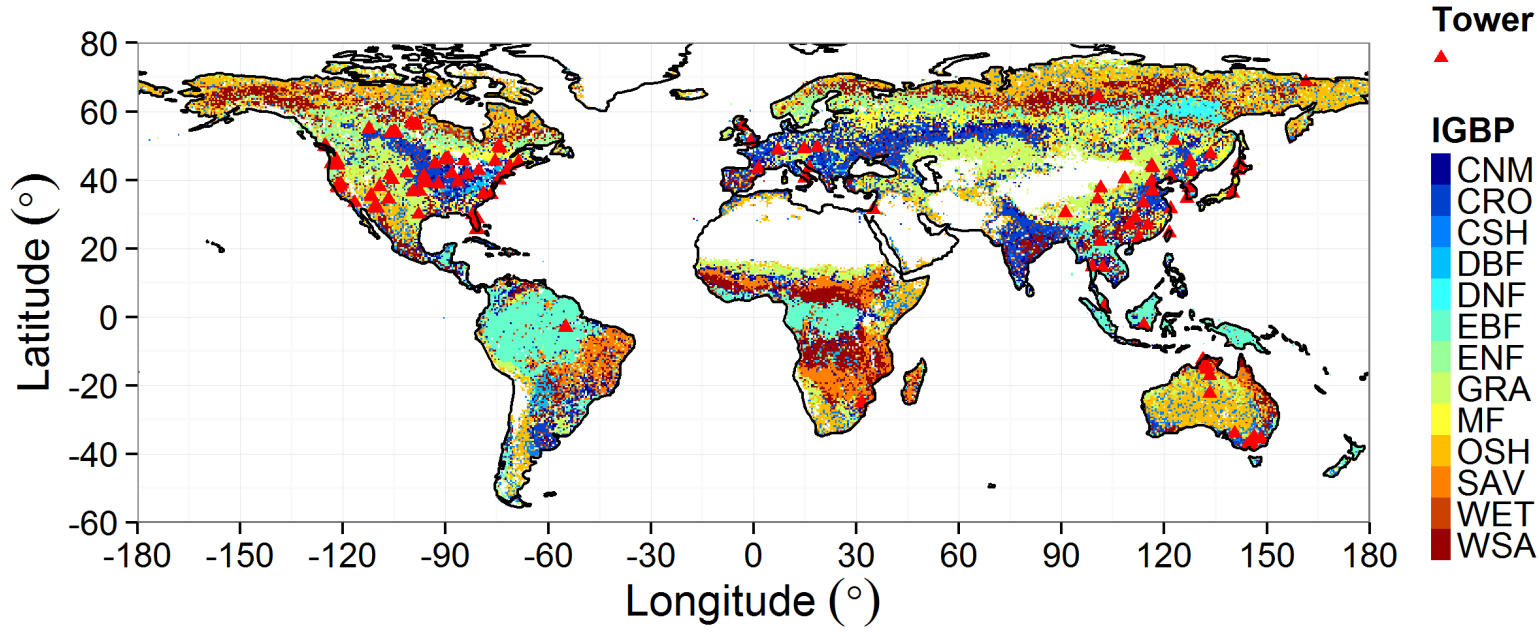

190

Fig. 1. Geographical distribution of flux towers overlaid onto the 2001 MODIS IGBP land cover map at a $0.5^{\circ} \times 0.5^{\circ}$ resolution.

\subsection{Benchmark dataset}

The model tree ensemble (MTE) approach was used to empirically up-scale FLUXNET measurements of fluxes (hereafter $\mathrm{GPP}_{\mathrm{MTE}}$ ) to the global scale. Explanatory variables for the model consisted of meteorological variables, the biophysical state of the vegetation, and vegetation types (Jung et al., 2009; Jung et al., 2011). GPP global FLUXNET up-scaling that has been used as a baseline for evaluating land surface models and estimating global $\mathrm{CO}_{2}$ uptake (e.g., Beer et al., 2010; Bonan et al., 2011). However, GPP ${ }_{\text {MTE }}$ has its own weakness, specifically for estimates of GPP in highproduction croplands (Guanter et al., 2014). Mean annual GPP $\mathrm{MTE}_{\mathrm{M}}$ was calculated at a spatial resolution of $0.5^{\circ}$ for the years $1982-2008$. The same grid was also applied in our global GPP estimation. 


\subsection{Satellite data}

207

208

209

210

211

212

213

214

215

216

217

218

219

220

221

222

\subsubsection{EVI data}

EVI is widely used as a proxy of canopy "greenness" to address spatial and temporal variations in terrestrial photosynthetic activity (e.g., Huete et al., 2002; Ma et al., 2013). EVI is defined as (Huete et al., 1997):

$$
E V I=G \frac{\rho_{\text {NIR }}-\rho_{\text {red }}}{\rho_{\text {NIR }}+C_{1} \times \rho_{\text {red }}-C_{2} \times \rho_{\text {blue }}+L}
$$

where $\rho_{N I R}, \rho_{\text {red }}$ and $\rho_{\text {blue }}$ are atmospherically corrected, either fully or partially, values of surface near-infrared (NIR, 841-876 nm), red (620-670 nm) and blue (459-479 nm) spectral reflectance, respectively; $G$ is the gain factor (set at 2.5); $L$ (set at 1.0 ) is the canopy background adjustment; and $C_{1}$ (set at 6 ) and $C_{2}$ (set at 7.5) are the coefficients of the aerosol resistance term, which uses the blue band to correct for the influence of aerosols in the red band.

MODIS monthly VI products (MOD13A3.005) for February 2000 to 2013 were obtained from the USGS repository (http://e4ft101.cr.usgs.gov/MOLT/MOD13A3.005/). This dataset is produced globally over land at $1-\mathrm{km}$ resolution and monthly compositing periods from atmospherically corrected surface reflectances. The compositing algorithm is based on a constrained-view angle-maximum value composite (CV-MVC) to minimize atmospheric and bidirectional reflectance distribution function (BRDF) influences (Huete et al., 2002).

It is difficult to precisely co-locate the pixels that directly correspond to the footprint of an EC tower (Sims et al., 2006b). Fluctuations in flux tower footprint size and shape, due to the underlying topography, vegetation, wind speed and etc., may induce a footprint mismatch between the tower and MODIS (Jung et al., 2009; Sims et al., 2006b; Sjöström et al., 2011). Where the landscape is homogenous, the scale mismatch is not a serious problem and the 
MODIS pixels can adequately represent flux site conditions. Discrepancies are typically observed at grassland and cropland sites, likely due to the fragmentation of these landscapes (Cescatti et al., 2012). However, sub- and inter-pixel heterogeneity is unavoidable in most cases and thus introduces additional bias. Consequently, a central $3 \times 3 \mathrm{~km}$ window surrounding the flux tower was used to extract mean EVI time series. The $3 \times 3 \mathrm{~km}$ window has been found to reduce scale mismatch relative to a centrally located $1 \mathrm{~km}$ pixel or window sizes of $5 \times 5$ or $7 \times 7 \mathrm{~km}$ (e.g., Ma et al., 2013; Rahman et al., 2005; Sims et al., 2006b; Sjöström et al., 2011; Xiao et al., 2005). At sites with spatially varying amounts of mixed vegetation types, averaging across the MODIS window is equivalent to averaging across time in flux measurements (Sims et al., 2006b).

\subsubsection{Smoothing method of EVI}

To reduce noise and uncertainties in the MODIS EVI time series at each site, the singular spectrum analysis (SSA) was employed. SSA is a data adaptive, non-parametric analysis approach based on embedding a time series $\{X(t): t=1, N\}$ in a vector space of dimension $M$ and it works well in the analysis of non-linear dynamics in geophysical datasets (Kondrashov and Ghil, 2006; Ma et al., 2013; Wang and Liang, 2008). The SSA technique consists of two complementary stages: decomposition and reconstruction (Hassani, 2007).

The one-dimensional time series $\{X(t): t=1, N\}$ is first embedded into a trajectory matrix $X=\left[X_{1}, \ldots X_{K}\right]=\left(x_{i j}\right)_{i, j=1}^{L, K}$, where $K=t-L+1$. Next, singular value decomposition (SVD) is applied to $X$ :

$$
X=\sum_{j=1}^{d} \sqrt{\lambda_{i}} U_{j} V_{j}^{T}, V_{j}=X^{\prime} / \sqrt{\lambda_{j}}
$$


251 where $\lambda_{j}$ is the jth eigenvalue of $X X^{\prime}, U_{j}$ is the jth eigenvector of $X X^{\prime}$, and $d$ is the rank of $252 X$. The reconstruction includes the eigentriple grouping and diagonal averaging (i.e., 253 Hankelization of a matrix), to produce a length $N$ time series from the matrix $X$. The SSA 254 method is more robust to outliers than linear filtering because it conducts a global 255 reconstruction (i.e., convolution) of the whole time series as with Fourier methods 256 (Alexandrov, 2009).

257 Key parameters in SSA are the decomposition window length $L$ and the number of leading components in reconstruction. In the monthly EVI time series, a window length of 37 (i.e. 37 months) and 6 leading components best captured the periodicity and simultaneously reduced random noises during reconstruction. The missing EVI value in January 2000 was extrapolated, thereby yielding a complete set of 14 years of EVI data. Since bare soil yields an EVI value of $0.08 \sim 0.10$, around which GPP is zero (Sims et al., 2008; Sims et al., 2006b), monthly values of EVI reconstructed from SSA were corrected by offsetting 0.10 to remove the background signal. EVI data are also contaminated by snow effects in mid- and high latitudes that result in a false positive signal (Huete et al., 2002). To minimise the contamination, the snow/ice flag in MOD13A3 VI quality assurance field was first used to remove the snow-covered EVI values; and then EVI values were further screened for effects of cold temperature (daytime land surface temperature below $-2{ }^{\circ} \mathrm{C}$; Tan et al., 2011; Zhang et al., 2004) using the MODIS land surface temperature product (MOD11C3). Finally, positive values of monthly EVI during non-snow and non-cold temperature periods were summed into annual, integrated values (iEVI) to regress against annual GPP calculated at each flux site. In our global GPP estimation, the $1 \mathrm{~km}$ EVI data were re-gridded into $0.5^{\circ}$ resolution to compare with global GPP $\mathrm{MTE}_{\mathrm{M}}$ and then processed the same way as the site level analysis. 


\subsubsection{Leaf area index data}

The MOD15A2.005 leaf area index (LAI) product is composited every 8 days at $1 \mathrm{~km}$ resolution and is available at the USGS repository (http://e4ft101.cr.usgs.gov/MOLT/MOD15A2.005). LAI is retrieved through a threedimensional radiative transfer model that requires land cover classification (Knyazikhin et al., 1998). By applying a procedure similar to that used for EVI, a central $3 \times 3 \mathrm{~km}$ window was used to extract LAI time series from 2000 to 2013, and SSA was applied to smooth the series. At each site, peak LAI values in individual site-years were averaged to represent the mean annual maximum LAI ( $\mathrm{LAI}_{\max }$ ) of the site and then the site LAI values within a land cover type were averaged to get the mean LAI for each of the land cover classifications except wetlands, which have no observed values in MOD15A2.005. In our analyses we assigned a mean LAI value of $6.3( \pm 2.3)$ for the 6 wetland sites, obtained from a global synthesis of LAI observations (Asner et al., 2003).

\subsubsection{Land cover types}

The MCD12Q1.005 land cover type product provides options of five global land cover classification systems. We used the IGBP land cover scheme which includes 17 land cover classes: water, evergreen needle-leaf forest (ENF), evergreen broadleaf forest (EBF), deciduous needle-leaf forest (DNF), deciduous broadleaf forest (DBF), mixed forest (MF), closed shrublands (CSH), open shrublands (OSH), woody savannas (WSA), savannas (SAV), grasslands (GRA), permanent wetlands (WET), croplands (CRO), urban and built-up, cropland/natural vegetation mosaic (CNM), snow and ice, barren or sparsely vegetated. The IGBP land cover map in 2001 was obtained from ORNL DAAC (http://webmap.ornl.gov/wcsdown/wcsdown.jsp?dg_id=10004_1). The product has a spatial 
resolution of $500 \mathrm{~m}$. In our global GPP estimation, this map was resampled into $0.5^{\circ}$

299

300

301

302

303

304

305

306

307

308

309

310

311

312 resolution to match the $\mathrm{GPP}_{\mathrm{MTE}}$ product. Four land cover types were excluded (water, urban and built-up, snow and ice, and barren or sparsely vegetated), and CNM was classed with

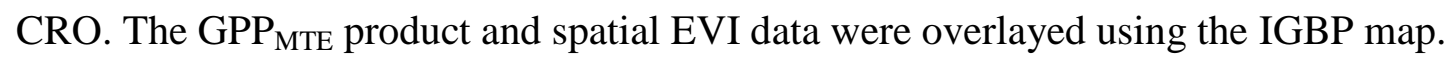

\subsection{Statistical analyses}

Annual GPP calculated for each site in each year was correlated with the corresponding annual iEVI using log-log regressions following (Campos et al., 2013). Since the goodnessof-fit for intra-annual GPP-EVI correlations may differ across biomes (Rahman et al., 2005; Sims et al., 2006b; Wu et al., 2010), the GPP-iEVI relationship was further investigated within each biome. The performance of these GPP-iEVI models within each biome was evaluated based on leave-one-out cross-validations (CV), which can test the practical accuracy of these models. The GPP-iEVI models based on biomes were then applied to the whole globe, and the final global GPP estimation was compared to GPP $\mathrm{MTE}_{\text {. Two standard }}$ statistical measures were employed to assess the regression relationships: the coefficient of determination $\left(R^{2}\right)$ and the root mean squared error (RMSE).

$$
\begin{gathered}
R^{2}=1-\frac{\sum_{i}\left(x_{i}-y_{i}\right)^{2}}{\sum_{i}\left(x_{i}-\bar{x}\right)^{2}} \\
R M S E=\sqrt{\frac{\sum_{i}\left(x_{i}-y_{i}\right)^{2}}{n}}
\end{gathered}
$$

where $x_{i}$ denotes the observed data, $y_{i}$ the modeled data, $\mathrm{n}$ the number of observations. $R^{2}$ represents the proportion of total variation of observed data explained by the model. RMSE measures the bias between modeled and observed data. 


\subsection{The overall relationship between GPP and iEVI without biomes categorization}

321 The significant logarithmic-logarithmic regression $\left(R^{2}=0.67, p<0.001\right.$, Fig. 2, inset)

322 between $\mathrm{GPP}_{\mathrm{EC}}$ and $\mathrm{iEVI}$ at the annual scale shows that there was a good general 323 correspondence between GPP $\mathrm{EC}_{\mathrm{C}}$ and iEVI across all biomes (Fig. 2). The leave-one-out cross-

324 validation based performance measures $\left(\mathrm{CV} R^{2}=0.66\right.$, Table 2$)$ further demonstrated the 325 effectiveness of the logarithmic model (log-log) in regressing GPP against iEVI. The

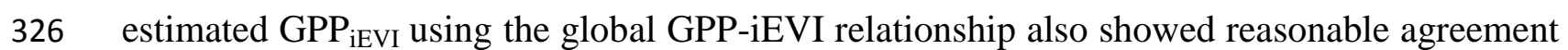
327 with $\operatorname{GPP}_{\mathrm{EC}}\left(R^{2}=0.62, p<0.001\right)$, although $\mathrm{GPP}_{\mathrm{iEVI}}$ was consistently underestimated 328 relative to field (EC) measurements. However, the point distribution in the relationship was 329 much more scattered at medium to high production biomes (approximately GPP $>800 \mathrm{~g} \mathrm{C} \mathrm{m}^{-}$ $\left.330{ }^{2} \mathrm{yr}^{-1}\right)$. The global relationship was unable to satisfactorily estimate GPP accurately for some 331 of the biomes, such as evergreen broadleaf forest and woody savannas, although the data 332 from these biomes still occurred within the overall distribution (Fig. 2). Consequently, it was 333 necessary to further investigate the individual GPP-iEVI relationship within each biome. 


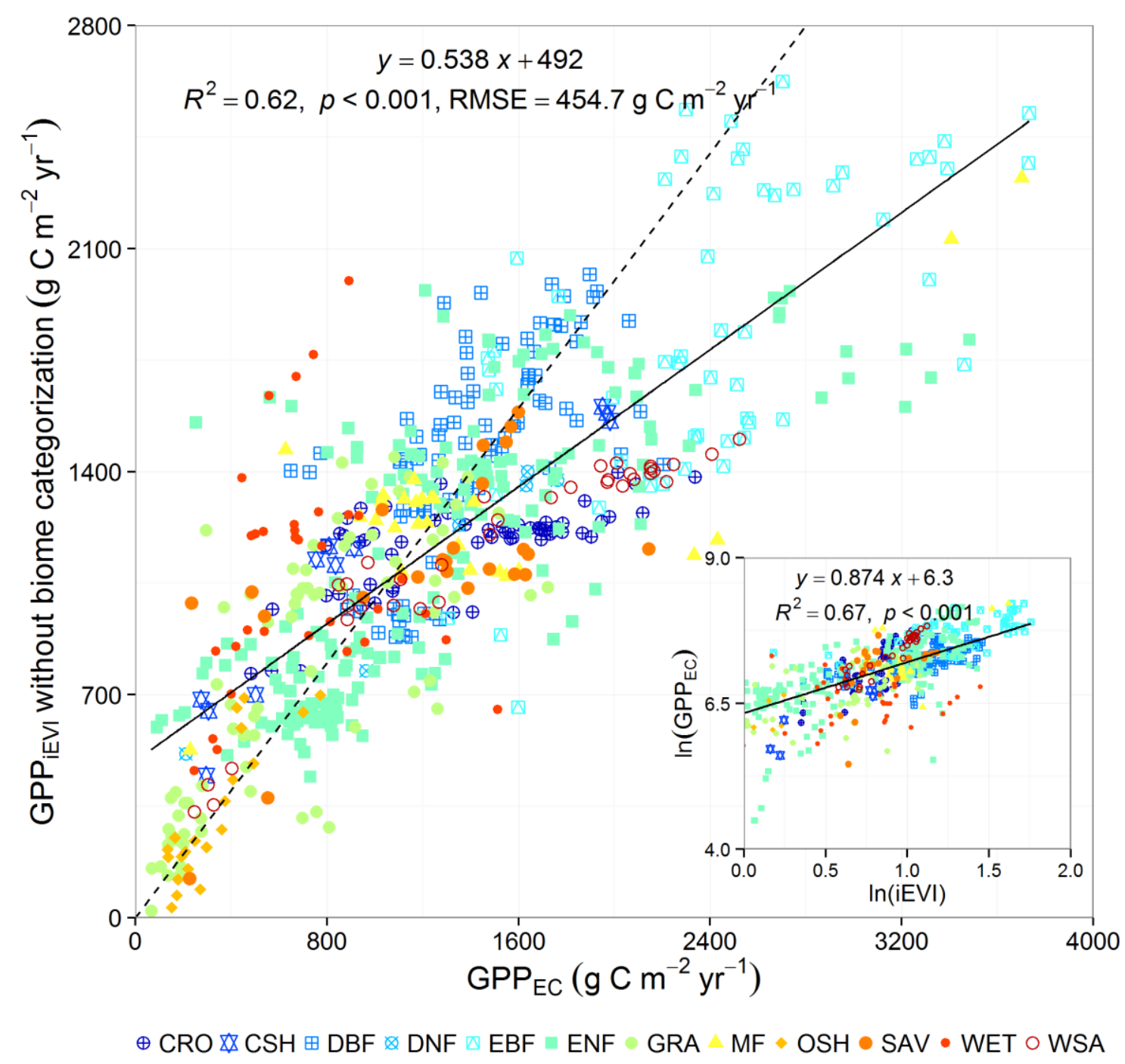

334

335 Fig. 2. Relationship between derived annual GPP from iEVI ( $\left.\mathrm{GPP}_{\mathrm{iEVI}}\right)$ and eddy covariance 336 tower measured GPP $\left(\mathrm{GPP}_{\mathrm{EC}}\right)$ since 2000 based on all sites and across 12 different biomes.

337 The inset shows $\ln$-transformed annual $\mathrm{GPP}_{\mathrm{EC}}$ and annual iEVI for all site-years. The solid 338 line represents the least squares regression line. The dashed line represents the 1:1 line.

\subsection{Biome-specific relationships between GPP and iEVI}

341 There was considerable variation among the 12 individual biome types in the regression

342 relationships between $\mathrm{GPP}_{\mathrm{EC}}$ and $\mathrm{iEVI}$ (Fig. 3). The poorest performance of iEVI in 
estimating $\mathrm{GPP}_{\mathrm{EC}}$ was observed in wetlands in raw regression $\left(R^{2}=0.11, p<0.05, \mathrm{RMSE}=\right.$ 297.2 $\mathrm{g} \mathrm{C} \mathrm{m}^{-2} \mathrm{yr}^{-1}$ ) and in cross validations (Table 2). The correlation was strongest in woody savannas, closed shrublands, deciduous needle-leaf forests, grasslands, and open shrublands; and moderate in croplands, evergreen needle-leaf forests, savannas, mixed forests, deciduous broadleaf forests, and evergreen broadleaf forests (Fig. 3). Meanwhile, we calculated the anomalies (by subtracting the mean value) of GPP and iEVI within each biome and investigated correlations between GPP anomaly and iEVI anomaly using linear regressions. Except for the wetlands, GPP anomaly and iEVI anomaly showed moderate to strong correlations within biomes (Figure S1). This result was consistent with our non-linear models using GPP and iEVI themselves (Figure 3). Further, cross-validations revealed that the GPPiEVI models within each biome (except wetlands) performed robustly and thus could be applied to the global scale. It is notable that the strength of the correlation within deciduous biomes was generally better (higher $R^{2}$ and lower RMSE) than those within evergreen vegetation, except deciduous broadleaf forest (still with better iEVI performance than evergreen broadleaf forest). The relationship for mixed forests had a similar $R^{2}$ to evergreen needle-leaf forest but was associated with the largest RMSE (490.3 $\mathrm{g} \mathrm{C} \mathrm{m}^{-2} \mathrm{yr}^{-1}$ ) across all biomes. There was also a wide range of values for the fitted slopes of the relationship between $\mathrm{GPP}_{\mathrm{EC}}$ and iEVI. Croplands and deciduous needle-leaf forests occupied a similar and narrow iEVI spectrum, but $\mathrm{GPP}_{\mathrm{EC}}$ of the former was more sensitive (larger slope) to iEVI. Among forested biomes, $\mathrm{GPP}_{\mathrm{EC}}$ of evergreen broadleaf forest was less sensitive to $\mathrm{iEVI}$ than evergreen needle-leaf forests. Likewise, $\mathrm{GPP}_{\mathrm{EC}}$ of deciduous broadleaf forests was less sensitive to $\mathrm{iEVI}$ than deciduous needle-leaf forests. $\mathrm{GPP}_{\mathrm{EC}}$ of grasslands and savannas, both of which are grass-dominated biomes, exhibited similar responses to iEVI. Although the range of $\mathrm{GPP}_{\mathrm{EC}}$ and $\mathrm{iEVI}$ values differed substantially among mixed forests and woody 


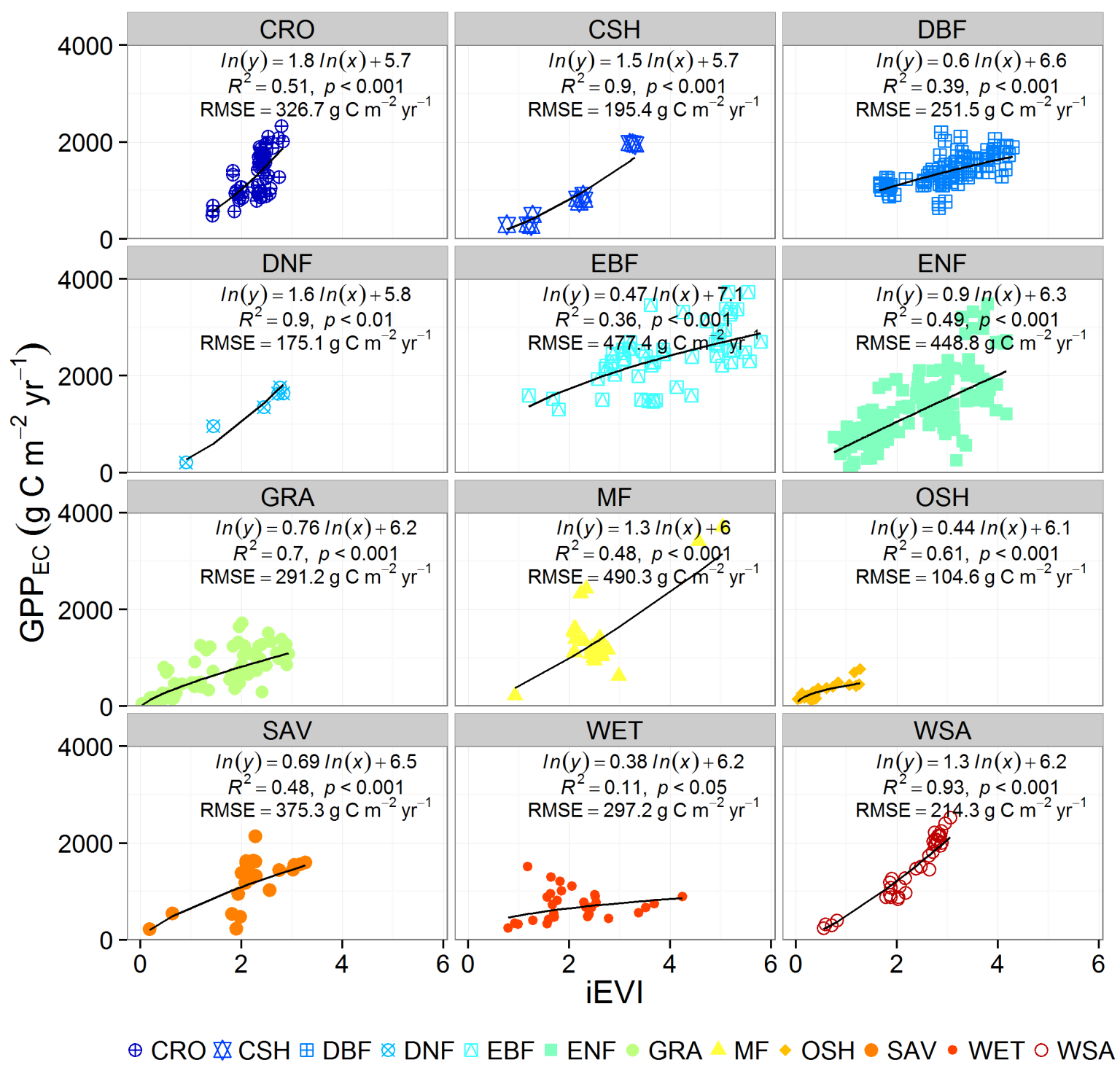

370 Fig. 3. Biome-specific relationships between tower-estimated annual GPP (GPP $\mathrm{EC}_{\text {) }}$ ) and iEVI.

371 Solid lines represent GPP-iEVI relationships derived from the $\ln \left(\mathrm{GPP}_{\mathrm{EC}}\right) \sim \ln (\mathrm{iEVI})$ formulas

372 within each biome. Coefficient of determination $\left(R^{2}\right)$ represents the fit goodness of the $373 \ln \left(\mathrm{GPP}_{\mathrm{EC}}\right) \sim \ln (\mathrm{iEVI})$ relationship, and the root mean squared error (RMSE) represents the 374 bias between GPP estimated using iEVI and GPP $_{\mathrm{EC}}$. All relationships are statistically 375 significant. 
Table 2. Summary of the raw and leave-one-out cross-validation (CV) performance measures

377 (coefficients of determination, $R^{2}$; root mean squared error, RMSE) of $\ln (\mathrm{GPP})-\ln (\mathrm{iEVI})$

378 models for all data points and each biome, respectively.

\begin{tabular}{ccccc}
\hline Biomes & Raw $R^{2}$ & CV $R^{2}$ & Raw RMSE & CV RMSE \\
\hline All & 0.67 & 0.66 & 454.7 & 455.8 \\
CRO & 0.51 & 0.48 & 326.7 & 335.9 \\
CSH & 0.90 & 0.81 & 195.4 & 239.6 \\
DBF & 0.39 & 0.36 & 251.5 & 256.0 \\
DNF & 0.90 & 0.51 & 175.1 & 284.9 \\
EBF & 0.36 & 0.32 & 477.4 & 492.1 \\
ENF & 0.49 & 0.48 & 448.8 & 454.2 \\
GRA & 0.70 & 0.67 & 291.2 & 296.2 \\
MF & 0.48 & 0.33 & 490.3 & 529.2 \\
OSH & 0.61 & 0.52 & 104.6 & 115.0 \\
SAV & 0.48 & 0.43 & 375.3 & 395.2 \\
WET & 0.11 & 0.01 & 297.2 & 311.9 \\
WSA & 0.93 & 0.93 & 214.3 & 222.8 \\
\hline
\end{tabular}

379

380 Estimated GPP based on the biome-specific GPP-iEVI formulae at all sites were then 381 compared with observed $\mathrm{GPP}_{\mathrm{EC}}$ (Fig. 4). The relationship between $\mathrm{GPP}_{\mathrm{iEVI}}$ and $\mathrm{GPP}_{\mathrm{EC}}$ was 382 significantly strengthened relative to the regression obtained without biome partitioning (Fig. 383 2) with increased $R^{2}$ (from 0.62 to 0.74 ), larger slope (from 0.538 to 0.723 ), decreased RMSE 384 (from 454.7 to $368.2 \mathrm{~g} \mathrm{C} \mathrm{m}^{-2} \mathrm{yr}^{-1}$ ) and smaller intercept (from 492 to 295). There was a large 385 dispersion of points around the linearly fitted function, but these points were mainly obtained 386 from high-GPP locations (> approximately $2400 \mathrm{~g} \mathrm{C} \mathrm{m}^{-2} \mathrm{yr}^{-1}$ ). 


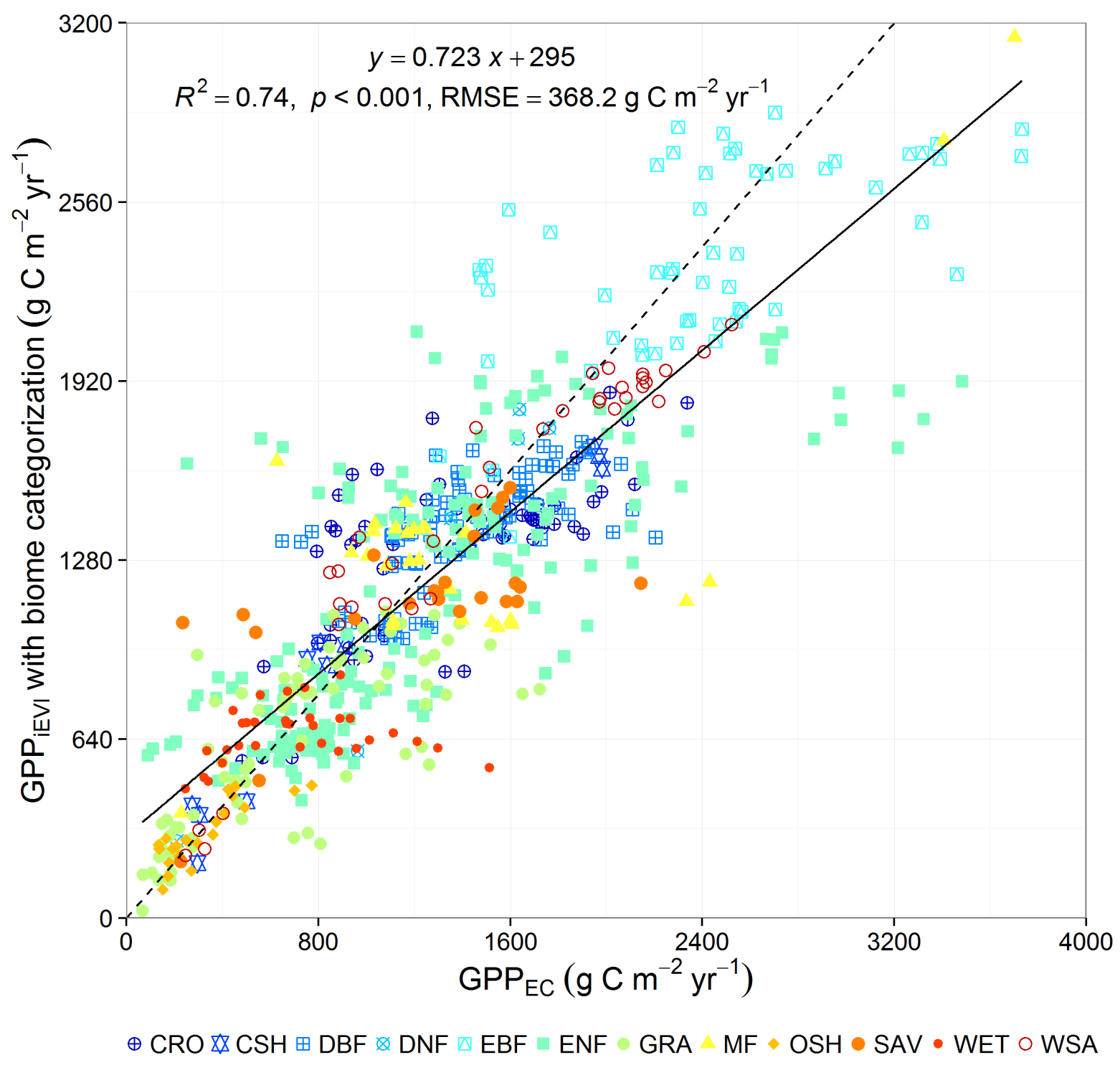

Fig. 4. Comparison of modelled annual GPP ( $\left.\mathrm{GPP}_{\mathrm{iEVI}}\right)$ using biome-specific GPP-iEVI relationships with eddy covariance tower measured GPP $\left(\mathrm{GPP}_{\mathrm{EC}}\right)$. The solid line represents the linear regression line. The dashed line represents the 1:1 line.

\subsection{Global application of biome-specific GPP-iEVI relationships}

The set of biome-specific GPP-iEVI relationships were applied to the global data of iEVI and IGBP land cover types. Per-pixel comparison between GPP $\mathrm{iEVI}_{\text {and }}$ aPP $\mathrm{MTE}_{\mathrm{MTE}}$ demonstrated the consistency of biome-specific GPP-iEVI models when up-scaling GPP from the site to global 
scales $\left(R^{2}=0.79, \mathrm{RMSE}=387.8 \mathrm{~g} \mathrm{C} \mathrm{m}^{-2} \mathrm{yr}^{-1}\right.$; Fig. 5). The very high accuracy of global, 397 multi-year, averaged GPP $\left(R^{2}=0.97\right)$ within each biome was unexpected given that the number of flux towers was restricted and their distribution was not geographically uniform.

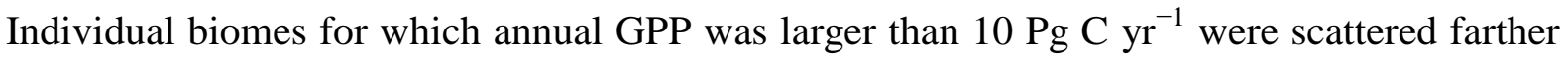
400 from the 1:1 line, resulting in underestimation of GPP by iEVI in EBF and overestimation in 401 WSA, CRO and CNM in comparison with the benchmark dataset.

402
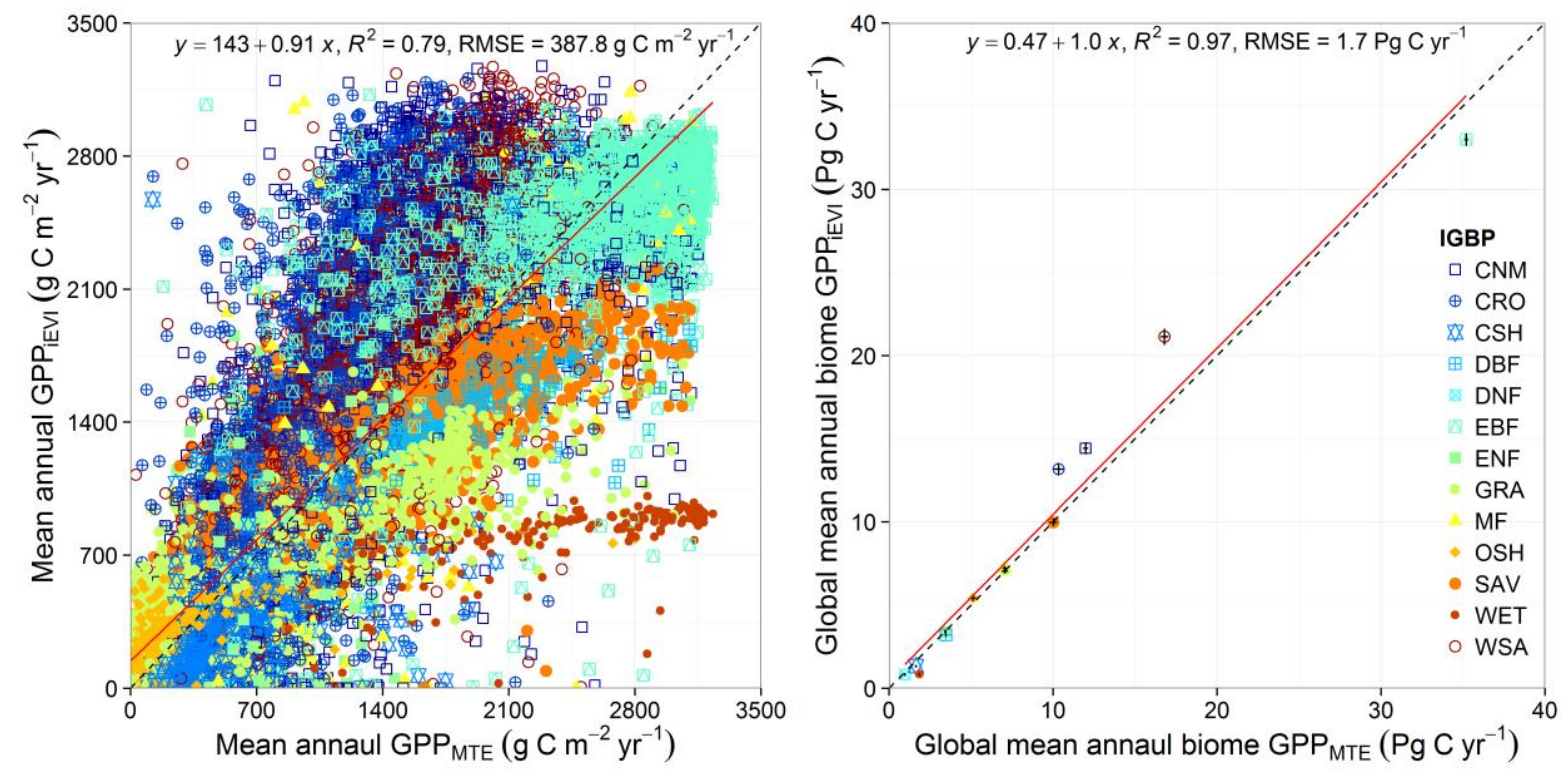

403

Fig. 5. Comparison between modelled average annual GPP using iEVI (GPP $\left.{ }_{\text {iEvI }}\right)(2000-2013)$

404

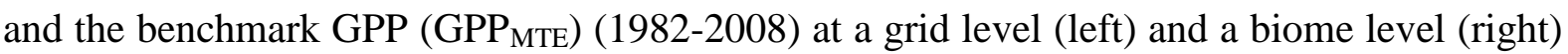
across the globe. The red solid line represents the linear regression line and the black dashed 406 line represents the 1:1 line. Horizontal and vertical error bars (right) indicate standard 407 deviations of mean annual biome GPP $\mathrm{iEVI}_{\mathrm{i}}$ (2000-2013) and GPP $\mathrm{MTE}_{\mathrm{M}}$ (1982-2008), 408 respectively. 

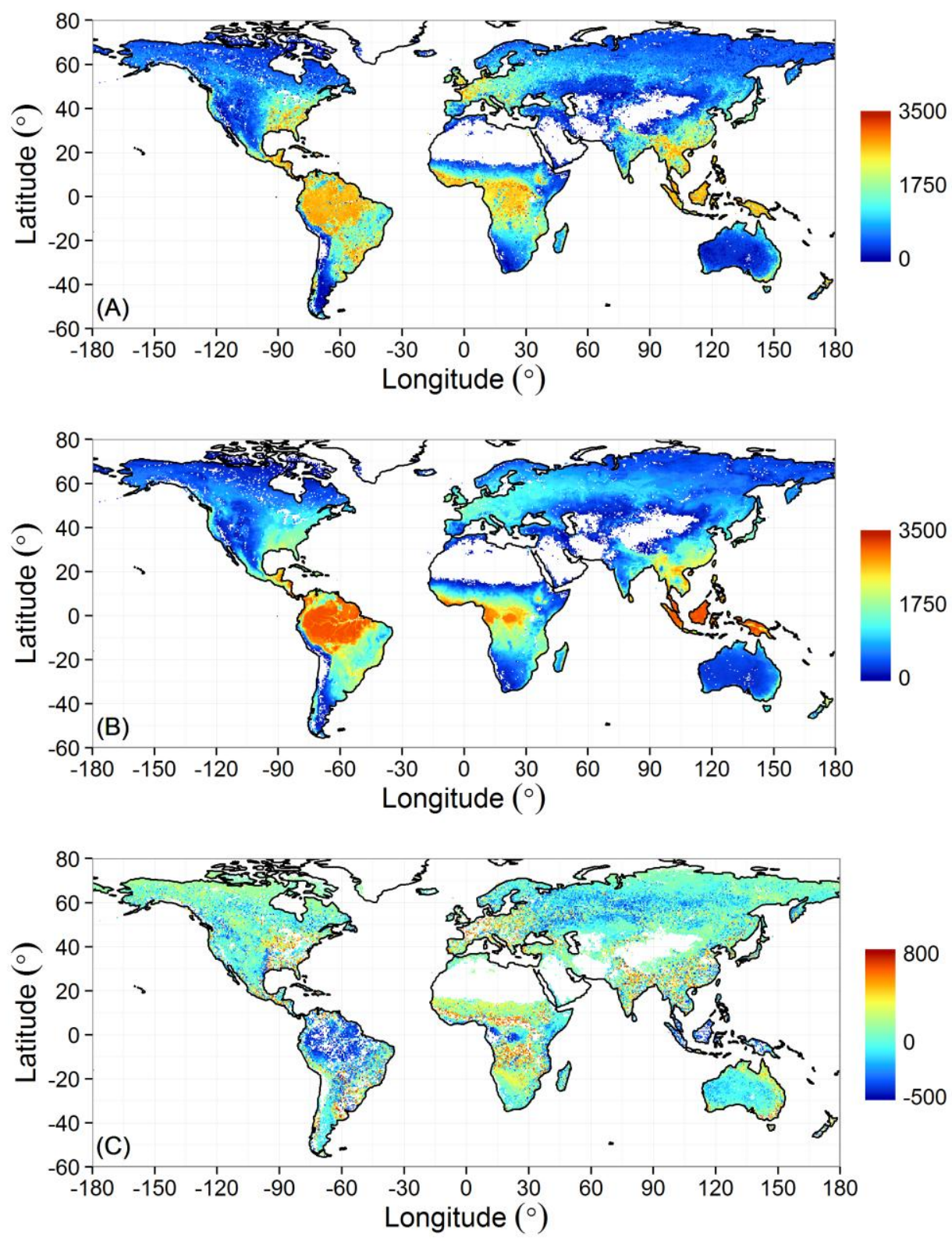

Fig. 6. Spatial comparison of (A) the mean annual GPP from iEVI (GPP $\left.{ }_{\text {iEVI, }} \mathrm{g} \mathrm{C} \mathrm{m}^{-2} \mathrm{yr}^{-1}\right)$

412 (2000-2013) with (B) the benchmark GPP (GPP $\left.\mathrm{MTE}, \mathrm{g} \mathrm{C} \mathrm{m}^{-2} \mathrm{yr}^{-1}\right)$ (1982-2008) and the 413 distribution of $(\mathrm{C})$ the residual $\left(\mathrm{g} \mathrm{C} \mathrm{m}^{-2} \mathrm{yr}^{-1}\right)$ between $\mathrm{GPP}_{\mathrm{iEVI}}$ and $\mathrm{GPP}_{\mathrm{MTE}}$ within the 5-95\% 414 quantile.

416 The mean spatial pattern of GPP was accurately reproduced by iEVI (Fig. 6). However, GPP 417 was primarily underestimated by iEVI in the tropics, western Russia and equatorial Africa 
and was overestimated in Europe, eastern North America, the high-latitude tropics of Africa,

419

420

421

422

423

424

425

426

$\mathrm{S}, 8^{\circ}-15^{\circ} \mathrm{N}, 20^{\circ}-28^{\circ} \mathrm{N}$ and $30^{\circ}-55^{\circ} \mathrm{N}$, and negative biases in the region $10^{\circ} \mathrm{S}-5^{\circ} \mathrm{N}$ (Fig. 7). southeastern South America, southeastern Australia, southeastern Asia, and parts of India and north China. In these regions, central Africa was dominated by tropical EBF (area around equator) and its north and south edges (area around $5^{\circ} \mathrm{N}$ and $5^{\circ} \mathrm{S}$ ) were dominated by woody savannas; Europe, eastern North America, southeastern South America and Australia, India and north China were widely covered by cropland/natural vegetation mosaics or croplands (Fig. 1), which were both parameterised as croplands when calculating $\mathrm{GPP}_{\mathrm{iEVI}}$. Latitudinal GPP derived from the iEVI showed positive biases from the benchmark in the regions $30^{\circ}-38^{\circ}$

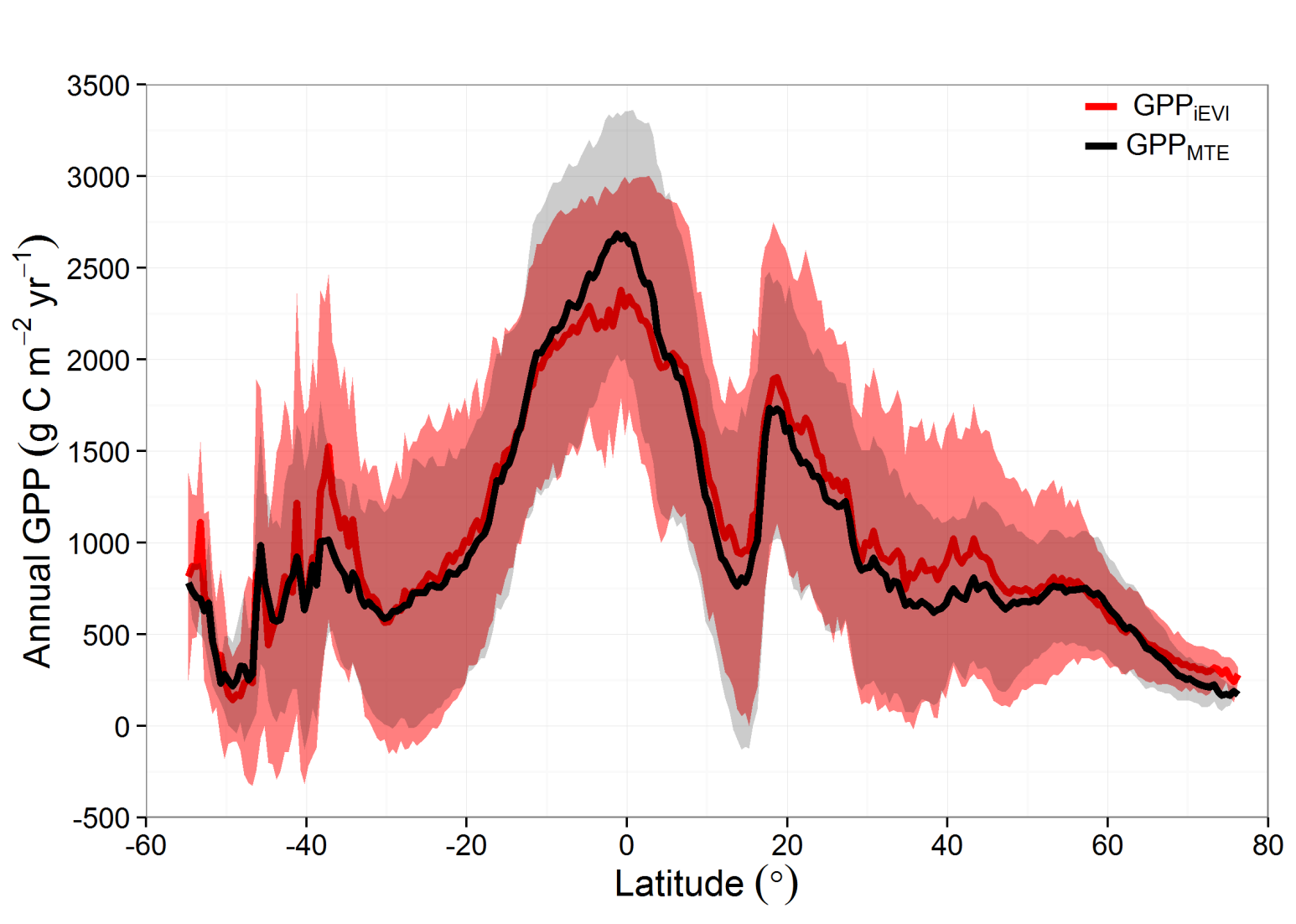

427

Fig. 7. Latitudinal patterns $\left(0.5^{\circ}\right.$ bands $)$ of mean annual GPP by iEVI (GPP $\left.{ }_{i E V I}, 2000-2013\right)$ and the benchmark (GPP $\left.\mathrm{GTE}_{\mathrm{MT}}, 1982-2008\right)$, respectively. The red shaded area represents the standard deviation of all GPP ${ }_{\text {iEvI }}$ values of cells along the latitude. The grey shaded area represents the standard deviation of all $\mathrm{GPP}_{\mathrm{MTE}}$ values of cells along the latitude. 


\subsection{Canopy structure effect on biome-specific GPP-iEVI relationships}

433

434

435

436

437

438

439

440

441

442

443

444

445

446

447

Among vegetation and climatic factors (mean annual maximal LAI, temperature, precipitation and VPD), only LAI and precipitation influenced the regression between GPP and iEVI. The strength of the biome-specific correlations between GPP and iEVI decreased with increasing mean annual LAI $\left(R^{2}=0.37, p<0.05\right)$ (Fig. 8). The strength of biomespecific GPP-iEVI relationships with mean annual precipitation followed the same negative trend, but the $R^{2}$ was only marginally statistically significant $\left(R^{2}=0.33, p=0.051\right.$; Fig. 8 inset). The slopes of relationships between $\mathrm{GPP}_{\mathrm{EC}}$ and $\mathrm{iEVI}$ (i.e., the sensitivity of GPP to EVI) across 12 biomes increased at small values of maximal LAI and then decreased across larger values of maximal LAI (LAI breakpoint was estimated to be $1.98 \mathrm{~m}^{2} \mathrm{~m}^{-2}$, Fig. 8).
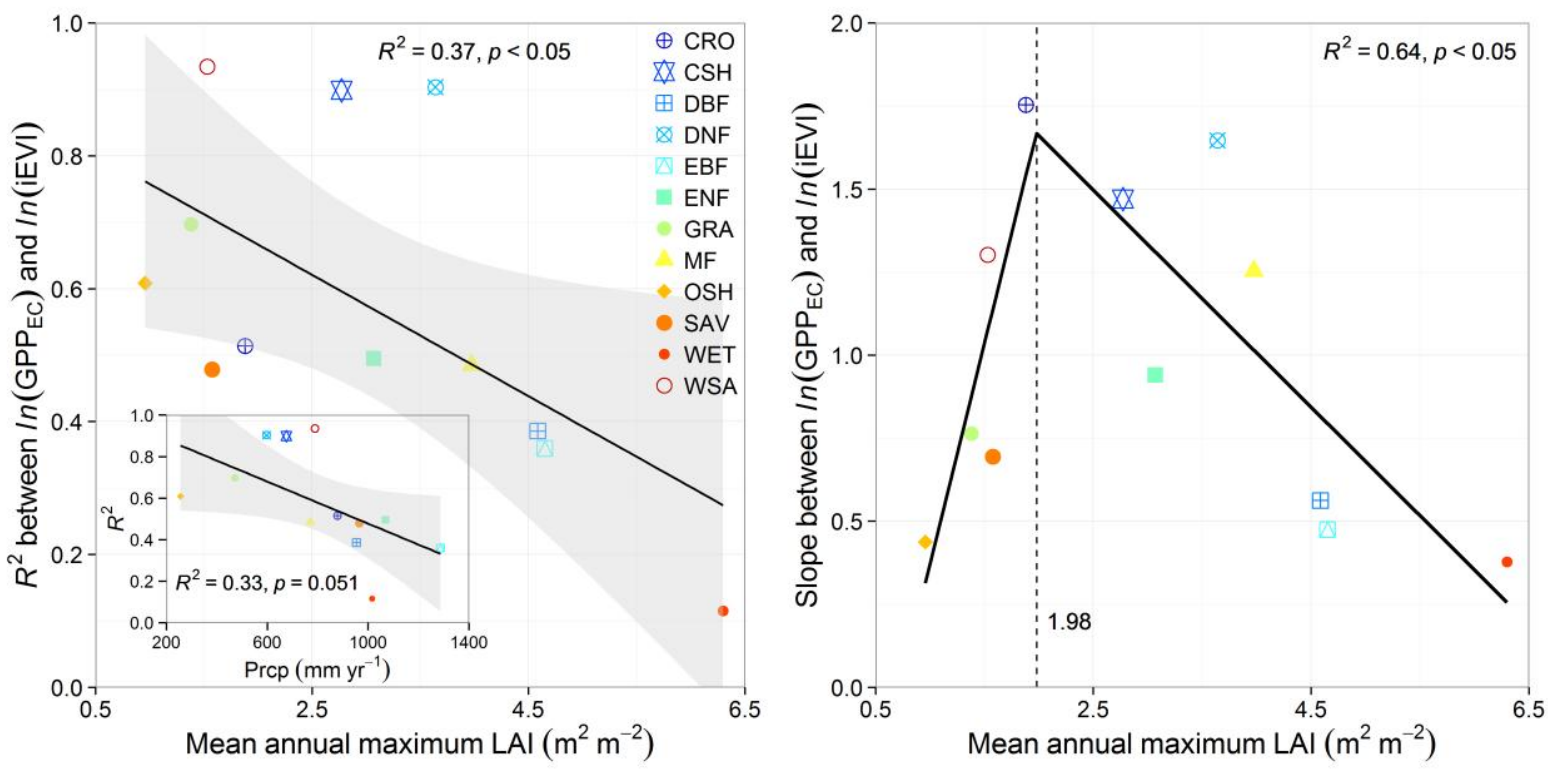

Fig. 8. Strength $\left(R^{2}\right)$ and slopes of relationships between $\ln \left(\mathrm{GPP}_{\mathrm{EC}}\right)$ and $\ln (\mathrm{iEVI})$ for each of all biomes as a function of either mean annual precipitation (Prcp, inset) or mean annual maximum LAI in each biome. Black lines represent the segmented linear regression line. The dashed vertical line indicates the breakpoint of the segmented linear relationship. The shaded area represents $95 \%$ confidence band. 


\subsection{Uncertainty analysis}

\subsubsection{Gap-filling and partitioning of eddy covariance carbon fluxes}

452 Two major concerns in up-scaling of eddy covariance measurements of fluxes are (1) the associated propagation of uncertainty within the source datasets, and (2) the up-scaling method itself. Systematic and random errors in measurement, gap-filling and partitioning procedures can result in uncertainty for estimates of GPP (Papale, 2006). Flux measurements can be subject to substantial random errors, which can be modelled as a double exponential distribution (Hollinger and Richardson, 2005). To minimise gap-filling errors in this study, we included only site-years without large gaps (less than $5 \%$ missing data). A short-term empirical temperature function was used to model ecosystem respiration in the MDS method and the robustness of this function depends on the noisiness of the flux data and the range of temperatures during the short period (Reichstein et al., 2005). Therefore, at sites with stable temperatures and noisy eddy covariance data, it can be difficult to establish a reliable relationship between ecosystem respiration and temperature (Reichstein et al., 2005). Consequently, datasets from the FLUXNET ANN product were preferred above the MDS product. The total annual error in eddy fluxes has been conservatively estimated to be below $200 \mathrm{~g} \mathrm{C} \mathrm{m}^{-2} \mathrm{yr}^{-1}$ (Reichstein et al., 2007) and the products from these standard methodologies are widely used in up-scaling and benchmarking models (e.g., Beer et al., 2010; Bonan et al., 2011; Jung et al., 2009; Rahman et al., 2005). However, it is noteworthy that neither the ANN nor the MDS method may be the best option in all flux sites. 


\subsubsection{Ecosystem heterogeneity}

472 A further source of error is introduced when scaling EVI to global GPP. To match the spatial resolution of $\mathrm{GPP}_{\mathrm{MTE}}$, MODIS EVI (1 km resolution) and IGBP classification maps $(500 \mathrm{~m}$ resolution) were resampled into $0.5^{\circ}$, thereby simplifying prediction of GPP at the global scale. However, resampling unavoidably introduced error in areas with mixed land cover types. The loss of information concerning landscape heterogeneity within larger pixels can cause misuse of the biome-specific GPP-iEVI formulae at the sub-pixel level. The incongruence between GPP ${ }_{\mathrm{iEVI}}$ and $\mathrm{GPP}_{\mathrm{MTE}}$ in WSA, CNM and CRO could be due to varying proportions of vegetation components within a grid cell. For WSA, the eddy covariance measured flux data were mostly from Australia, where the woody and herbaceous components of woody savannas are substantially heterogeneous (Hirota et al., 2011). Even within a single continent (e.g., Africa or Australia), woody savannas display significant variation in structural and phenological patterns (Kutsch et al., 2008; Sjöström et al., 2011; Sjöström et al., 2013). However, EVI only has a moderate capacity to predict ecosystem structural and functional attributes such as basal cover of vegetated patches, perennial plants species richness and retention of nutrients (Gaitán et al., 2013). Similar situations (e.g., different species, cultivars and fragments of croplands) can be encountered in CNM and CRO, besides the fact that the CRO specific GPP-iEVI formula was applied in CNM. In addition, crops are generally intensely managed (e.g., irrigation, fertilisation, sowing and harvest), which constrains the reflectance-based greenness indices to accurately estimate GPP of crops (Guanter et al., 2014). Consequently, the benchmark dataset GPP MTE $_{\text {underestimates cropland }}$ GPP in large agricultural regions such as the US Corn Belt, the Indo-Gangetic Plain and the North China Plain but tends to moderately overestimate cropland GPP in South America (Guanter et al., 2014). Thus, the overestimation of $\mathrm{GPP}_{\mathrm{iEVI}}$ in comparison with $\mathrm{GPP}_{\mathrm{MTE}}$ in croplands of North America, north India and north China (Fig. 6) seems reasonable but is still 
biased in other agricultural areas. Limitations arising from global scaling can be overcome

497

498

499

500

501

502

503

504

505

506

507

508

509

510

511

512

513

514

515

516

517

518

519

520

using the original relatively high-spatial resolution satellite data (Sjöström et al., 2011; Zhao

et al., 2005). However, using these fine resolution data will inevitably increase modeling complexity. Furthermore, the $500 \mathrm{~m}$ resolution MODIS IGBP map has its own weakness and uncertainty (Friedl et al., 2010). Errors due to global scaling were similar in GPP $_{\text {iEVI }}$ and $\mathrm{GPP}_{\mathrm{MTE}}$ and consequently were comparable in this study (Fig. 5).

\subsection{The relationships between EVI and GPP}

Vegetation greenness indices (VIs) associated GPP models are generally based on one of the following two hypothetical relationships between either LUE or GPP and VIs. The first holds that VIs provide proxy information for parameterizing LUE or fAPAR (Gitelson et al., 2006; Inoue et al., 2008; Sims et al., 2006a; Sims et al., 2006b; Wu et al., 2012) in photosynthetic (as opposed to non-photosynthetic) tissues (Xiao et al., 2004a; Xiao et al., 2004b; Xiao et al., 2005). Following the logic of classical LUE theory, various LUE models have been developed based on eddy covariance observation and satellite data (Gitelson et al., 2006; Peng et al., 2011; Running et al., 2004; Sims et al., 2008; Yuan et al., 2010). Each of these models includes a combination of equations that are scaled by environmental regulation of GPP (Beer et al., 2010). The second is that VIs can estimate GPP alone. Values of EVI follow changes in the greenness and structure of vegetation regardless of the cause of those variations (Huete et al., 2002), resulting in a stronger correlation between tower-estimated GPP and EVI than the correlations between tower-estimated GPP and MODIS GPP or between tower LUE and EVI during the photosynthetic period (Sims et al., 2006b). The assumption that EVI can be taken as a proxy of LUE results in curvilinear relationships between GPP and EVI (Sims et al., 2006a). This strongly supports our results (Fig. 3), suggesting the two hypotheses are essentially consistent, and it is therefore reasonable to 
assume a strong correlation between GPP and EVI. However, EVI does not perform

522

523

524 satisfactorily across all vegetation types, particularly at evergreen forest sites (Rahman et al., 2005; Sims et al., 2006b; Wu et al., 2010). Furthermore, EVI is not able to capture GPP variations at short time-scales because short-term fluctuations in photosynthetic capacity are not reflected by variations in canopy greenness over physiological timescales (Sims et al., 2006a). For example, low temperature can significantly and rapidly reduce GPP whilst having little effect on canopy greenness (Wu et al., 2010).

\subsection{Environmental constraints on the ability of EVI to estimate GPP}

\subsubsection{LAI affects covariation $\left(R^{2}\right)$ of GPP with iEVI}

The performance of EVI in estimating GPP is constrained by environmental conditions, including features of both climate and vegetation structure. The covariation between GPP and EVI is often better in deciduous sites than evergreen sites (Rahman et al., 2005; Sims et al., 2006b; Wu et al., 2010). Deciduous sites experience a large range between maximal and minimal EVI (as a result of large seasonal variation) and among sites the range is significantly correlated with mean summer rainfall (positive correlation) or mean summer VPD (negative correlation) (Sims et al., 2006b). Our results showed that the strength of the correlation between GPP and iEVI in deciduous biomes was generally better than in evergreen biomes, although the strength of the correlation in DBF was only slightly better than in EBF. Evergreen biomes show smaller seasonal variation in EVI than deciduous biomes. Observable seasonal variation in vegetation greenness may be a prerequisite for successful use of VIs to estimate vegetation production. Deciduous biomes demonstrate distinct seasonal dynamics of leaf greenness, thus satellite data can accurately capture these large seasonal changes in greenness (Ma et al., 2013; Verma et al., 2014). In contrast, it is 
difficult to achieve the same level of accuracy within evergreen biomes. This presumably explains the poor performance of iEVI in estimating GPP of EBF, either in wet tropical areas

547

(Fig. 5, 6, 7) or in semi-arid evergreen forests, where photosynthetic capacity can vary independently of EVI and LAI in response to dry conditions (Maseyk et al., 2008). However, either the seasonal change of EVI or LAI cannot effectively explain the weak correlation between iEVI and GPP in DBF. Nagai et al. (2010) found EVI to increase earlier than GPP during the leaf-expansion period in DBF, and this caused systematic variability in the GPPEVI relationship (Richardson et al., 2012; Verma et al., 2014). To address the asynchronicity between GPP and EVI in DBF, a phenological scalar may be needed in GPP-iEVI equations, as has been applied in the vegetation photosynthesis model of Xiao et al. (2004b). This suggests that large seasonal variance of EVI does not necessarily imply a good correlation of EVI and GPP and thus EVI variance is not appropriate to explain the covariation of iEVIGPP across biomes. Our result also showed that iEVI variance across biomes can be greatly divergent while $R^{2}$ of iEVI-GPP correlations can be close. For example, iEVI standard deviations for CRO and ENF are 0.44 and 0.94 , whereas R2 are 0.51 and 0.49 , respectively (Figure 3). In contrast, peak LAI can be as a metric of the complexity of canopy structures of a biome and thus is appropriate to indicate the covariation strength of iEVI-GPP relationships. As peak LAI increases, the iEVI-GPP relationship is weakened (Fig. 8) by the increased structure complexity due to either small seasonal EVI variations in a biome such as EBF or the asynchronicity between GPP and EVI in a biome such as DBF.

Another possible factor contributing to the poor correlation between GPP and iEVI in vegetation with high LAI that are most located in wet regions may be the extensive cloudy conditions that reduce the quality of EVI retrievals (Nagai et al., 2010). In arid and semi-arid areas where cloud cover is minimal, precipitation is a controlling factor of vegetation phenology and productivity (Bradley et al., 2011; Cleverly et al., 2013; Huxman et al., 2004; 
Jolly and Running, 2004; Ma et al., 2013; Schwinning and Sala, 2004). Moreover, peak LAI is typically limited by water availability in arid and semi-arid regions (Eamus and Prior, 2001; Sjöström et al., 2011). Consequently, the correlation between the strength of GPP-iEVI relationships with mean annual precipitation showed the same trend as that for $\mathrm{LAI}_{\max }$ (Fig. 8 inset). The correlation between the strength of GPP-iEVI relationships and $\mathrm{LAI}_{\max }$ may help identifying the regions where iEVI is most likely to be a good predictor of GPP. Globally, underestimation of GPP in some locations was compensated by overestimation in other locations within the same biome type (Fig. 5, 6), with a consequential minimisation of biases on the estimation of GPP due to global patterns of LAI.

\subsubsection{LAI scales the sensitivity (fitted slopes) of GPP to iEVI}

Lindroth et al. (2008) proposed that LAI is the principal scaling parameter for GPP in northern deciduous and coniferous forests. In biomes with a relatively small peak LAI (e.g., less than $2.5 \mathrm{~m}^{2} \mathrm{~m}^{-2}$ ), the sensitivity of GPP to EVI increases with LAI (Sjöström et al., 2011), although there were too few arid vegetation classes in the study to identify a statistically significant trend in sensitivity across small values of LAI (less than $1.98 \mathrm{~m}^{2} \mathrm{~m}^{-2}$, Fig. 8). Conversely, as vegetation become less water limited, the sensitivity of GPP to EVI tended to decrease across large values of LAI (larger than $1.98 \mathrm{~m}^{2} \mathrm{~m}^{-2}$, Fig. 8), which to our knowledge has not yet been found at a global scale. Degradation of the GPP-iEVI relationship at large LAI is due to (1) decreased sensitivity of variation in EVI to changes in canopy structure, including LAI and canopy type, of dense forests (Gao et al., 2000) and (2) biased or decreased seasonality of variations in EVI. 


\section{Conclusions}

594

595

596

597

598

599

600

601

602

603

604

605

606

607

608

609

610

611

612

613

614

615

616

We comprehensively evaluated the ability of MODIS EVI to estimate annual GPP across 12 land cover types based on GPP from eddy covariance. iEVI does not require calculation of the duration of the growing season, which significantly simplifies the estimation of annual GPP by EVI at the global scale. Cross validations demonstrated the robustness of biomespecific $\ln (\mathrm{GPP}) \sim \ln (\mathrm{iEVI})$ models. In comparison to a global benchmarking dataset of mean annual GPP, we showed that the performance of iEVI was consistent from site to global scales. Compared to $\mathrm{GPP}_{\mathrm{MTE}}, \mathrm{GPP}_{\mathrm{iEVI}}$ performed better in croplands of high productivity but poorer mainly in forests. The strength of the GPP-iEVI relationships across biomes was correlated with peak LAI, by which the slope was also scaled. These findings suggest that vegetation structure is an important factor regulating the accuracy and sensitivity of EVI in estimating spatial patterns of annual GPP across multiple biomes. While LUE models, datadriven models and terrestrial biosphere models are usually difficult to parameterize or are limited by coarse resolution meteorological inputs, our study provides a promising and very convenient approach to estimate global spatial patterns of GPP at either a fine or coarse resolution. Nevertheless, the use of EVI in estimating GPP requires further study, especially in deciduous broadleaf forest and evergreen biomes. Our findings on impacts of vegetation structure provide valuable information for such efforts in improving EVI-based models of GPP.

\section{Acknowledgements}

This research was supported by an Australian Research Council Discovery Early Career Research Award (project number DE120103022). X.T. was supported by one of National Basic Research Program of China (grant number 2013CB733404) and the National Natural 
Science Foundation of China (grant number 41101379). This work used eddy covariance data acquired by the FLUXNET community and in particular by the following networks: AmeriFlux (U.S. Department of Energy, Biological and Environmental Research, Terrestrial Carbon Program (DE-FG02-04ER63917 and DE-FG02-04ER63911)), AfriFlux, AsiaFlux, CarboAfrica, CarboEuropeIP, CarboItaly, CarboMont, ChinaFlux, FLUXNET Canada (supported by CFCAS, NSERC, BIOCAP, Environment Canada, and NRCan), GreenGrass, KoFlux, LBA, NECC, OzFlux, TCOS - Siberia, USCCC. We acknowledge the financial support to the eddy covariance data harmonization provided by CarboEuropeIP, FAO-GTOSTCO, iLEAPS, Max Planck Institute for Biogeochemistry, National Science Foundation, University of Tuscia, Université Laval, Environment Canada and U.S. Department of Energy and the database development and technical support from Berkeley Water Center, Lawrence Berkeley National Laboratory, Microsoft Research eScience, Oak Ridge National Laboratory, University of California Berkeley, University of Virginia.

\section{References}

Alexandrov, T., 2009. A method of trend extraction using singular spectrum analysis. RevStat, 7: 1-22.

Asner, G.P., Scurlock, J.M. and A Hicke, J., 2003. Global synthesis of leaf area index observations: implications for ecological and remote sensing studies. Global Ecology and Biogeography, 12(3): 191-205.

Aubinet, M. et al., 2000. Estimates of the annual net carbon and water exchange of forests: the EUROFLUX methodology. Advances in ecological research, 30: 113-175.

Baldocchi, D., 2008. 'Breathing' of the terrestrial biosphere: lessons learned from a global network of carbon dioxide flux measurement systems. Australian Journal of Botany, 56(1): 1-26.

Baldocchi, D. et al., 2001. FLUXNET: A new tool to study the temporal and spatial variability of ecosystem-scale carbon dioxide, water vapor, and energy flux densities. Bulletin of the American Meteorological Society, 82(11): 2415-2434. 
Beer, C. et al., 2010. Terrestrial gross carbon dioxide uptake: global distribution and covariation with climate. Science, 329(5993): 834-838.

Bonan, G.B. et al., 2011. Improving canopy processes in the Community Land Model version 4 (CLM4) using global flux fields empirically inferred from FLUXNET data. Journal of Geophysical Research: Biogeosciences (2005-2012), 116(G2).

Bradley, A.V. et al., 2011. Relationships between phenology, radiation and precipitation in the Amazon region. Global Change Biology, 17(6): 2245-2260.

Campos, G.E.P. et al., 2013. Ecosystem resilience despite large-scale altered hydroclimatic conditions. Nature, 494(7437): 349-352.

Cescatti, A. et al., 2012. Intercomparison of MODIS albedo retrievals and in situ measurements across the global FLUXNET network. Remote sensing of environment, 121: 323-334.

Ciais, P. et al., 2005. Europe-wide reduction in primary productivity caused by the heat and drought in 2003. Nature, 437(7058): 529-533.

Cleverly, J. et al., 2013. Dynamics of component carbon fluxes in a semi-arid Acacia woodland, central Australia. Journal of Geophysical Research: Biogeosciences, 118: $1168-1185$.

Eamus, D. and Prior, L., 2001. Ecophysiology of trees of seasonally dry tropics: Comparisons among phenologies, Advances in ecological research. Academic Press, pp. 113-197.

Friedl, M.A. et al., 2010. MODIS Collection 5 global land cover: Algorithm refinements and characterization of new datasets. Remote Sensing of Environment, 114(1): 168-182.

Gaitán, J.J. et al., 2013. Evaluating the performance of multiple remote sensing indices to predict the spatial variability of ecosystem structure and functioning in Patagonian steppes. Ecological Indicators, 34: 181-191.

Gao, X., Huete, A.R., Ni, W. and Miura, T., 2000. Optical-biophysical relationships of vegetation spectra without background contamination. Remote Sensing of Environment, 74(3): 609-620.

Gitelson, A.A. et al., 2006. Relationship between gross primary production and chlorophyll content in crops: Implications for the synoptic monitoring of vegetation productivity. Journal of Geophysical Research: Atmospheres (1984-2012), 111(D8).

Goward, S.N. and Huemmrich, K.F., 1992. Vegetation canopy PAR absorptance and the normalized difference vegetation index: an assessment using the SAIL model. Remote Sensing of Environment, 39(2): 119-140. 
678

679

680

681

682

683

684

685

686

687

688

689

690

691

692

693

694

695

696

697

698

699

700

701

702

703

704

705

706

707

708

709

710

711

Guanter, L. et al., 2014. Global and time-resolved monitoring of crop photosynthesis with chlorophyll fluorescence. Proceedings of the National Academy of Sciences, 111(14): E1327-E1333.

Hassani, H., 2007. Singular spectrum analysis: methodology and comparison.

Heinsch, F.A. et al., 2006. Evaluation of remote sensing based terrestrial productivity from MODIS using regional tower eddy flux network observations. Geoscience and Remote Sensing, IEEE Transactions on, 44(7): 1908-1925.

Hirota, M., Holmgren, M., Van Nes, E.H. and Scheffer, M., 2011. Global resilience of tropical forest and savanna to critical transitions. Science, 334(6053): 232-235.

Hollinger, D. and Richardson, A., 2005. Uncertainty in eddy covariance measurements and its application to physiological models. Tree Physiology, 25(7): 873-885.

Huete, A. et al., 2002. Overview of the radiometric and biophysical performance of the MODIS vegetation indices. Remote Sensing of Environment, 83(1): 195-213.

Huete, A., Liu, H., Batchily, K. and Van Leeuwen, W., 1997. A comparison of vegetation indices over a global set of TM images for EOS-MODIS. Remote sensing of environment, 59(3): 440-451.

Huxman, T.E. et al., 2004. Precipitation pulses and carbon fluxes in semiarid and arid ecosystems. Oecologia, 141(2): 254-268.

Inoue, Y., Peñuelas, J., Miyata, A. and Mano, M., 2008. Normalized difference spectral indices for estimating photosynthetic efficiency and capacity at a canopy scale derived from hyperspectral and $\mathrm{CO}_{2}$ flux measurements in rice. Remote Sensing of Environment, 112(1): 156-172.

Jolly, W.M. and Running, S.W., 2004. Effects of precipitation and soil water potential on drought deciduous phenology in the Kalahari. Global Change Biology, 10(3): 303-308.

Jung, M., Reichstein, M. and Bondeau, A., 2009. Towards global empirical upscaling of FLUXNET eddy covariance observations: validation of a model tree ensemble approach using a biosphere model. Biogeosciences, 6(10).

Jung, M. et al., 2011. Global patterns of land-atmosphere fluxes of carbon dioxide, latent heat, and sensible heat derived from eddy covariance, satellite, and meteorological observations. Journal of Geophysical Research: Biogeosciences (2005-2012), 116(G3).

Jung, M. et al., 2008. Diagnostic assessment of European gross primary production. Global Change Biology, 14(10): 2349-2364.

Knyazikhin, Y., Martonchik, J., Myneni, R., Diner, D. and Running, S., 1998. Synergistic algorithm for estimating vegetation canopy leaf area index and fraction of absorbed 
photosynthetically active radiation from MODIS and MISR data. Journal of Geophysical Research: Atmospheres (1984-2012), 103(D24): 32257-32275.

Kondrashov, D. and Ghil, M., 2006. Spatio-temporal filling of missing points in geophysical data sets. Nonlinear Processes in Geophysics, 13(2): 151-159.

Kutsch, W. et al., 2008. Response of carbon fluxes to water relations in a savanna ecosystem in South Africa. Biogeosciences Discussions, 5(3).

Lindroth, A. et al., 2008. Leaf area index is the principal scaling parameter for both gross photosynthesis and ecosystem respiration of Northern deciduous and coniferous forests. Tellus B, 60(2): 129-142.

Ma, X. et al., 2013. Spatial patterns and temporal dynamics in savanna vegetation phenology across the North Australian Tropical Transect. Remote Sensing of Environment, 139: 97 115.

Mao, D., Wang, Z., Li, L. and Ma, W., 2014. Spatiotemporal dynamics of grassland aboveground net primary productivity and its association with climatic pattern and changes in Northern China. Ecological Indicators, 41: 40-48.

Maseyk, K.S. et al., 2008. Physiology-phenology interactions in a productive semi - arid pine forest. New Phytologist, 178(3): 603-616.

Melillo, J.M. et al., 1993. Global climate change and terrestrial net primary production. Nature, 363(6426): 234-240.

Monteith, J., 1972. Solar radiation and productivity in tropical ecosystems. Journal of applied ecology, 9(3): 747-766.

Nagai, S., Saigusa, N., Muraoka, H. and Nasahara, K.N., 2010. What makes the satellitebased EVI-GPP relationship unclear in a deciduous broad-leaved forest? Ecological Research, 25(2): 359-365.

Papale, D., 2006. Towards a standardized processing of Net Ecosystem Exchange measured with eddy covariance technique: algorithms and uncertainty estimation.

Papale, D. and Valentini, R., 2003. A new assessment of European forests carbon exchanges by eddy fluxes and artificial neural network spatialization. Global Change Biology, 9(4): $525-535$.

Peng, Y., Gitelson, A.A., Keydan, G., Rundquist, D.C. and Moses, W., 2011. Remote estimation of gross primary production in maize and support for a new paradigm based on total crop chlorophyll content. Remote Sensing of Environment, 115(4): 978-989. 
Rahman, A., Sims, D., Cordova, V. and El-Masri, B., 2005. Potential of MODIS EVI and surface temperature for directly estimating per-pixel ecosystem C fluxes. Geophysical Research Letters, 32(19): L19404.

Reichstein, M. et al., 2005. On the separation of net ecosystem exchange into assimilation and ecosystem respiration: review and improved algorithm. Global Change Biology, 11(9): 1424-1439.

Reichstein, M. et al., 2007. Determinants of terrestrial ecosystem carbon balance inferred from European eddy covariance flux sites. Geophysical Research Letters, 34(1).

Richardson, A.D. et al., 2012. Terrestrial biosphere models need better representation of vegetation phenology: results from the North American Carbon Program Site Synthesis. Global Change Biology, 18(2): 566-584.

Running, S.W. et al., 2004. A continuous satellite-derived measure of global terrestrial primary production. Bioscience, 54(6): 547-560.

Schwinning, S. and Sala, O.E., 2004. Hierarchy of responses to resource pulses in arid and semi-arid ecosystems. Oecologia, 141(2): 211-220.

Sims, D.A. et al., 2006a. Parallel adjustments in vegetation greenness and ecosystem $\mathrm{CO}_{2}$ exchange in response to drought in a Southern California chaparral ecosystem. Remote Sensing of Environment, 103(3): 289-303.

Sims, D.A. et al., 2008. A new model of gross primary productivity for North American ecosystems based solely on the enhanced vegetation index and land surface temperature from MODIS. Remote Sensing of Environment, 112(4): 1633-1646.

Sims, D.A. et al., 2006b. On the use of MODIS EVI to assess gross primary productivity of North American ecosystems. Journal of Geophysical Research: Biogeosciences, 111(G4): G04015.

Sjöström, M. et al., 2011. Exploring the potential of MODIS EVI for modeling gross primary production across African ecosystems. Remote Sensing of Environment, 115(4): 10811089.

Sjöström, M. et al., 2013. Evaluation of MODIS gross primary productivity for Africa using eddy covariance data. Remote sensing of environment, 131: 275-286.

Tan, B. et al., 2011. An enhanced TIMESAT algorithm for estimating vegetation phenology metrics from MODIS data. Selected Topics in Applied Earth Observations and Remote Sensing, IEEE Journal of, 4(2): 361-371. 
Verma, M. et al., 2014. Remote sensing of annual terrestrial gross primary productivity from MODIS: an assessment using the FLUXNET La Thuile data set. Biogeosciences, 11(8): 2185-2200.

Wang, D. and Liang, S., 2008. Singular Spectrum Analysis for Filling Gaps and Reducing Uncertainties of MODIS Land Products, Geoscience and Remote Sensing Symposium, 2008. IGARSS 2008. IEEE International. IEEE, pp. 558-561.

Wang, K. and Dickinson, R.E., 2012. A review of global terrestrial evapotranspiration: Observation, modeling, climatology, and climatic variability. Reviews of Geophysics, 50(2): RG2005.

Wu, C., Munger, J.W., Niu, Z. and Kuang, D., 2010. Comparison of multiple models for estimating gross primary production using MODIS and eddy covariance data in Harvard Forest. Remote Sensing of Environment, 114(12): 2925-2939.

Wu, C., Niu, Z. and Gao, S., 2012. The potential of the satellite derived green chlorophyll index for estimating midday light use efficiency in maize, coniferous forest and grassland. Ecological Indicators, 14(1): 66-73.

Wylie, B.K. et al., 2003. Calibration of remotely sensed, coarse resolution NDVI to $\mathrm{CO}_{2}$ fluxes in a sagebrush-steppe ecosystem. Remote Sensing of Environment, 85(2): 243-255.

Xiao, X. et al., 2004a. Satellite-based modeling of gross primary production in an evergreen needleleaf forest. Remote sensing of environment, 89(4): 519-534.

Xiao, X. et al., 2004b. Modeling gross primary production of temperate deciduous broadleaf forest using satellite images and climate data. Remote Sensing of Environment, 91(2): 256-270.

Xiao, X., Zhang, Q., Hollinger, D., Aber, J. and Moore III, B., 2005. Modeling gross primary production of an evergreen needleleaf forest using MODIS and climate data. Ecological Applications, 15(3): 954-969.

Yang, F. et al., 2007. Developing a continental-scale measure of gross primary production by combining MODIS and AmeriFlux data through Support Vector Machine approach. Remote Sensing of Environment, 110(1): 109-122.

Yuan, W. et al., 2010. Global estimates of evapotranspiration and gross primary production based on MODIS and global meteorology data. Remote Sensing of Environment, 114(7): 1416-1431.

Zhang, Q. et al., 2005. Estimating light absorption by chlorophyll, leaf and canopy in a deciduous broadleaf forest using MODIS data and a radiative transfer model. Remote Sensing of Environment, 99(3): 357-371. 
810

811

812

813

814

815

816

Zhang, X., Friedl, M.A., Schaaf, C.B. and Strahler, A.H., 2004. Climate controls on vegetation phenological patterns in northern mid - and high latitudes inferred from MODIS data. Global Change Biology, 10(7): 1133-1145.

Zhao, M., Heinsch, F.A., Nemani, R.R. and Running, S.W., 2005. Improvements of the MODIS terrestrial gross and net primary production global data set. Remote sensing of Environment, 95(2): 164-176. 